ISSN 0103-5150

Fisioter. Mov., Curitiba, v. 23, n. 2, p. 183-192, abr./jun. 2010

Licenciado sob uma Licença Creative Commons

\title{
Análise do treinamento proprioceptivo no equilíbrio de atletas de futsal feminino
}

\author{
Analysis of proprioceptive training in the balance of women's futsal athletes
}

\author{
Fábio Oliveira Baldaço ${ }^{[a]}$, Vinícius Piccoli Cadó ${ }^{[b]}$, Jaqueline de Souza ${ }^{[c]}$, \\ Carlos Bolli Mota ${ }^{[\mathrm{d}]}$, Jadir Camargo Lemos ${ }^{[\mathrm{e}]}$
}

[a] Acadêmico do Curso de Fisioterapia, Universidade Federal de Santa Maria, Santa Maria, RS - Brasil, e-mail: fabio_baldaco@ yahoo.com.br

[b] Acadêmico do Curso de Fisioterapia, Universidade Federal de Santa Maria, Santa Maria, RS - Brasil, e-mail: viniciuscado@ hotmail.com

[c] Mestre, professora da Universidade Federal do Pampa, Uruguaiana, RS - Brasil, e-mail: jaquelinesz@yahoo.com.br

[d] Doutor, professor do Curso de Educação Física da Universidade Federal de Santa Maria, Santa Maria, RS - Brasil, e-mail: bolli@ cefd.ufsm.br

[e] Doutor, professor do Curso de Fisioterapia da Universidade Federal de Santa Maria, Santa Maria, RS - Brasil, e-mail: jadir.lemos@ gmail.com

\section{Resumo}

Objetivo: O objetivo deste trabalho foi avaliar a eficiência de um protocolo de exercícios proprioceptivos no equilíbrio de atletas de futsal feminino. Metodologia: A amostra foi composta por cinco atletas do time de futsal da Universidade Federal de Santa Maria (UFSM). Os treinamentos ocorreram três vezes por semana, durante um mês. As atletas foram avaliadas pré e pós-treinamento em uma plataforma de força no Laboratório de Biomecânica do Centro de Educação Física e Desportos da UFSM. Resultados: Os resultados mostraram uma redução estatisticamente significativa na média e na amplitude do centro de pressão pré e pós-intervenção, na direção médio-lateral e condição olhos fechados, o que demonstra que houve maior controle do equilíbrio corporal na posição e condição solicitada. Não foram encontradas diferenças estatisticamente significativas nas variáveis do centro depressão na condição olhos abertos. Acredita-se que esse resultado esteja relacionado à dominância da aferência visual sobre a proprioceptiva, que pode ter interferido na avaliação das alterações proprioceptivas. Conclusão: $O$ protocolo de propriocepção sugerido demonstrou maior controle de equilíbrio postural nas condições e na amostra avaliada.

Palavras-chave: Propriocepção. Futsal. Equilíbrio. 


\begin{abstract}
Objective: The objective of this study was to evaluate the efficiency of a proprioceptive exercise protocol in the balance of women's futsal athletes. Methodology: The sample was composed by five athletes from the futsal team of Universidade Federal de Santa Maria (UFSM). The training took place three times a week for one month. The athletes were evaluated pre and post-training on a strength platform in the Biomechanics Laboratory of the Physical Education and Sports Center of UFSM. Results: The results showed a statistically significant reduction in the average and in the amplitude of the pressure center, pre and post-intervention, in medium-lateral direction and in eye-closed condition, showing that there was a greater control of the body balance in the position and condition requested. There were no statistically significant differences in the pressure center and eye-open condition variables. It is believed that this result is related to the dominance of the visual afference on the proprioceptive one, which may have interfered in the evaluation of the proprioceptive alterations. Conclusion: The proprioceptive exercise protocol showed a greater control of the body balance in the condition and sample evaluated.
\end{abstract}

Keywords: Proprioception. Futsal. Balance.

\title{
Introdução
}

A prática do futebol de salão feminino foi autorizada pela Federação Internacional de Futebol de Salão (FIFUSA) em 23 de abril de 1983. Desde então se nota grande crescimento na quantidade de atletas desta modalidade, acompanhado de um avanço no número de competições, tornando-se um esporte profissional no País (1). Entretanto, embora seja visto um maior número de adeptas da prática desta modalidade, a figura da mulher no futebol ainda é tímida, seja por fatores relacionados à questão cultural brasileira (e à ideia do futebol como um "esporte masculino"), seja pela falta de incentivo e patrocínio das equipes profissionais de futebol feminino (2).

O futebol como modalidade esportiva está associado a um alto índice de lesões corporais, causadas pelos padrões de movimentos exigidos, como saltos, corridas curtas e longas, mudanças rápidas de direção, cabeceios, chutes e muito contato físico entre os jogadores $(1,3,4)$. Dentre as lesões ocorridas em atletas de futebol estão, principalmente, a entorse, a fratura-luxação, as lesões musculares e tendíneas $(3,5,6)$. Além das características próprias desta modalidade, outros fatores podem contribuir para a ocorrência de lesões, como alterações posturais, baixos índices de flexibilidade, movimentos desportivos incorretos, equipamentos inadequados, traumas diretos e o déficit proprioceptivo nos membros inferiores, principalmente nas articulações de joelho e tornozelo $(3,5,7,8)$.

A propriocepção é um mecanismo de percepção corporal em que os receptores periféricos (localizados em estruturas como músculos, tendões e articulações) enviam informações relativas ao movimento, estado de posição ou grau de deformação gerado nestas estruturas ao sistema nervoso central (SNC), que terá a função de processar, organizar e comandar o corpo adequadamente a fim de manter o controle postural $(9,10,11)$. Juntamente com a propriocepção, outros dois sistemas, igualmente importantes para a manutenção do equilíbrio, estão atuando: o sistema visual e o vestibular (10). O equilíbrio, por sua vez, é um processo dinâmico da postura corporal para prevenir quedas, pela manutenção da projeção do centro de gravidade dentro da área da base de suporte do corpo, que requer ajustes constantes da atividade muscular e do posicionamento articular, baseado nas informações enviadas pela visão, sistema vestibular e sistema proprioceptivo $(9,10)$.

O comprometimento do sistema proprioceptivo acarreta déficits na estabilização articular neuromuscular, que pode contribuir para a ocorrência de lesões como distensão excessiva das cápsulas e ligamentos articulares e, consequentemente, para a desestabilização postural (11). Nos atletas futebolistas, o déficit proprioceptivo é observado principalmente nas lesões articulares de tornozelo, causados por mecanismos comuns como a entorse ou por contusões em traumas diretos por outro adversário (12).

Muitos estudos têm mostrado que programas de exercícios que estimulam as vias sensoriais proprioceptivas podem vir a melhorar a estabilidade do equilíbrio, reduzindo a incidência de lesões nos esportes (13-17). Os exercícios proprioceptivos demonstram uma grande ação profilática e de reabilitação em lesões 
musculoesqueléticas, pois exigem da modalidade sensorial uma forma mais competente para obtenção de informações referentes à sensação de movimento e posição articular, com base em elementos de outras fontes que não a visual, a auditiva ou a cutânea superficial (18).

Os atletas de futebol, homens ou mulheres, estão expostos a um alto risco de injúrias que levam ao afastamento da prática da modalidade, perda do condicionamento físico e, algumas vezes, ao afastamento total do esporte (6). Estudos investigativos buscam alternativas que visem a reduzir o risco de injúrias e/ou melhorar o condicionamento e reabilitação desses desportistas $(7,16,19)$. Nessa linha, o presente estudo teve como objetivo avaliar a aplicação de um protocolo proprioceptivo no equilíbrio postural de atletas de futsal feminino.

\section{Materiais e métodos}

Este estudo caracteriza-se como do tipo quase-experimental, composto por um único grupo submetido à intervenção. Previamente à sua execução, o projeto de pesquisa foi submetido à avaliação do Comitê de Ética da Universidade Federal de Santa Maria (UFSM), que aprovou sua realização de acordo com as diretrizes estabelecidas na Resolução 196/96 do Conselho Nacional de Saúde, segundo Certificado de Apresentação para Apreciação Ética n. 0043.0.243.000-07.

A amostra do estudo foi composta pelas atletas integrantes do time de futsal feminino da UFSM, em Santa Maria, RS. Consentiram em participar do estudo, por meio de assinatura do Termo de Consentimento Livre Esclarecido, 12 atletas que preencheram os critérios de inclusão em entrevista prévia (integrantes do time de futsal, sem presença de lesões em membro inferior nos seis meses precedentes à pesquisa). No decorrer da aplicação do protocolo proprioceptivo proposto, sete sujeitos foram excluídos da amostra pela indisponibilidade de tempo para a realização dos treinos. Sendo assim, a amostra final ficou composta por cinco atletas.

Inicialmente foi aplicado um questionário adaptado do estudo de Bonetti e Coelho (7) para obtenção de informações referentes à dominância do membro inferior, histórico de lesões, conhecimento sobre propriocepção e tempo de prática do futsal. A seguir, foram obtidos os dados referentes às características físicas das atletas antes e após a aplicação do protocolo. O peso e a estatura foram verificados com uma balança e um estadiômetro da marca Welmy ${ }^{\mathbb{R}}$. Os resultados da caracterização da amostra encontram-se na Tabela 1.

Tabela 1 - Características físicas da amostra e sessões de treino proprioceptivo

\begin{tabular}{lcc}
\hline & Média & Desvio Padrão \\
\hline Idade (anos) & 20,6 & 0,8 \\
Peso antes (kg) & 60,36 & 2,55 \\
Altura (metros) & 1,67 & 0,03 \\
Peso depois (kg) & 60,86 & 2,79 \\
Sessões (número) & 10,6 & 1,74 \\
\hline
\end{tabular}

Nota: Para a análise do equilíbrio postural foi utilizada uma Plataforma de Força (OR6-5).

AMTI (Advanced Mechanical Technology, Inc.) é um instrumento que afere sinais de força e momentos durante o posicionamento do indivíduo sobre ele. Através dos sinais detectados por esse equipamento, é possível calcular os deslocamentos do centro de pressão (COP) nas direções ântero-posterior [COPy $=(\mathrm{Mx}-(\mathrm{Fy}) / \mathrm{Fz})]$ e médio-lateral $[\mathrm{COPx}=(\mathrm{My}-(\mathrm{Fx}) / \mathrm{Fz})]$. O estudo do COP está relacionado com o 
controle neuromuscular necessário para a manutenção do equilíbrio na postura em pé (9). No presente estudo este instrumento foi utilizado para informar acerca do controle e estabilidade postural estática unipodal após a aplicação de um protocolo de propriocepção. Segundo a bibliografia, a oscilação do centro de pressão é a principal medida, na postura estática, para mensurar o equilíbrio corporal $(9,20)$. As mudanças ocorridas no centro de gravidade durante o movimento ou a oscilação corporal são refletidas nas alterações observadas no centro de pressão e, consequentemente, informam acerca do equilíbrio ou estabilidade corporal (21).

O posicionamento, a orientação e a coleta de dados foram realizados individualmente, com roupas confortáveis, os pés descalços e nas condições olhos abertos e olhos fechados. As participantes foram orientadas a subir na plataforma e permanecer na postura em pé, com apoio unipodal sobre o membro inferior não dominante, braços ao longo do corpo, e com membro inferior dominante em flexão de joelho de aproximadamente $90^{\circ}$, o mais imóvel possível, por 40 segundos. A coleta de dados foi realizada com uma frequência de amostragem de $100 \mathrm{~Hz}$.

O processamento dos dados da plataforma foi realizado, primeiramente, através da filtragem das forças e momentos, por um filtro Butterworth, de ordem $3(22,23)$ com frequência de corte de $6 \mathrm{~Hz}$, definido segundo procedimento de Análise Residual, proposto por Winter (24). Em seguida foram calculados os deslocamentos do COP nas direções ântero-posterior (COPy) e médio-lateral (COPx). As variáveis analisadas dos deslocamentos do COP foram: média de deslocamento, desvio padrão e amplitude de deslocamento (subtração dos valores máximos e mínimos), em ambas as direções.

Para a análise estatística das variáveis do COP foi realizada pelo teste T de Student a verificação de distribuição e normalidade dos dados. Em vista do resultado da ausência de normalidade, utilizou-se o teste de comparações de Wilcoxon para dados não paramétricos com significância de 5\%. O programa estatístico utilizado foi o Estatistical Analisys System ${ }^{\circledR}$, Versão 8.02.

\section{Protocolo de treinamento}

O protocolo foi elaborado a partir de exercícios proprioceptivos sugeridos por Araújo et al. (18), Salgado (25), Prentice (26), Kisner e Colby (27), Bonetti e Coelho (7), Sheth et al. (12) e Bahr et al. (13). O treinamento proprioceptivo foi realizado três vezes por semana, em grupo, sob orientação dos pesquisadores, totalizando uma média de 10,5 (8-13) sessões com duração de 40 minutos.

As sessões iniciavam com uma sequência de alongamentos estáticos e seguiam com a aplicação dos exercícios, descritos a seguir:

\section{Exercícios grau um:}

- atleta caminhava sobre uma linha de 10 metros desenhada no chão. Com um pé em frente ao outro;

- caminhada com a distância de 10 metros sobre diferentes planos intercalados (colchonetes, piso e travesseiros), utilizando os seguintes apoios: calcanhar, ponta dos pés e bordas lateral e medial dos pés.

\section{Exercícios grau dois:}

- com apoio unipodal, a atleta passava objetos para a companheira de trás, girando apenas o tronco, sustentando-se primeiro com a perna direita e depois com a esquerda;

- as atletas executaram um total de cinco agachamentos por série com braços à frente e o apoio de um pé só; a outra perna permanecia em flexão de quadril e joelho.

\section{Exercícios grau três:}

- em apoio bipodal e joelhos estendidos, as atletas buscavam manter o equilíbrio na prancha e no disco de equilíbrio;

- a atleta executava um agachamento com os braços à frente e com o apoio de um pé só sobre o balancim (evolução: execução com olhos fechados); 
- a participante pulava na cama elástica e realizava deslocamentos laterais, para frente e para trás.

\section{Exercícios grau quatro:}

- usando o balancim, atleta com os olhos fechados tentava manter o equilíbrio enquanto desenhava um círculo em um local pré-definido;

- marcha sobre diferentes apoios (balancim, prancha, colchonete, cama elástica e demarcação no chão);

- as atletas pulavam em uma cama elástica, fazendo passes laterais (evolução: troca dos passes por "cabeceio" na bola);

- atletas apoiadas na prancha, ou no disco, ou ainda pulando na cama elástica, tentavam aparar a bola jogada pelos pesquisadores.

Os exercícios foram aplicados de maneira progressiva, conforme a evolução apresentada por cada atleta, e a cada sessão as participantes eram reavaliadas, mediante a observação da execução da sequência dos exercícios prescritos. A avaliação para a progressão foi realizada subjetivamente, pelo tempo em que as atletas mantinham as posições (ideal de 50 segundos) e pelo relato durante a prática do exercício proposto (exercício difícil de executar, médio e fácil). Quando o exercício tornava-se fácil e as atletas não apresentavam dificuldades para sua execução, estavam aptas para passar ao grau seguinte.

\section{Resultados e discussão}

Os resultados referentes ao questionário aplicado encontram-se na Tabela 2.

Tabela 2 - Resultados do Questionário adaptado de Bonetti e Coelho (7)

\begin{tabular}{cccc}
\hline Sujeito & Dominância & Tempo de prática do futsal & Conhecimento sobre propriocepção \\
\hline 1 & direita & 8 anos & já ouviu falar \\
2 & direita & 10 anos & já ouviu falar \\
3 & direita & 10 anos & sim \\
4 & direita & 12 anos & já ouviu falar \\
5 & direita & 6 anos & já ouviu falar \\
\hline
\end{tabular}

$\mathrm{Na}$ amostra, a dominância para chute foi encontrada como predominantemente destra e o tempo médio de prática do futsal foi 9,2 anos. Embora as atletas praticassem essa modalidade já há algum tempo, a grande maioria delas não possuía conhecimento sobre propriocepção. A falta de conhecimento a respeito da propriocepção revela a ausência de técnicas ou métodos que incorporem o trabalho proprioceptivo no treino da modalidade.

Os resultados dos deslocamentos do COP em ambas as direções e condições, pré e pós-treino proprioceptivo, são apresentados nas Tabela 3, 4, 5 e 6.

Na situação olhos fechados na direção médio-lateral (COPx), as atletas apresentaram uma redução estatisticamente significativa das variáveis média e amplitude de deslocamento do COPx. Da mesma forma, o desvio padrão também mostrou uma redução em seu valor, embora não comprovado estatisticamente, na comparação pré e pós-aplicação do protocolo. Esses resultados apontam para uma redução e consequente melhora no controle e estabilidade postural das atletas observados nesta direção. 
Tabela 3 - Dados refentes aos deslocamentos médio-laterais do centro de pressão (COPx) na condição - olhos fechados e apoio unipodal antes e após intervenção

\begin{tabular}{|c|c|c|c|c|c|c|}
\hline \multirow[b]{2}{*}{ Sujeito } & \multicolumn{3}{|c|}{ Pré-intervenção } & \multicolumn{3}{|c|}{ Pós-intervenção } \\
\hline & Média (m) & Amplitude (m) & $d p$ & Média (m) & Amplitude (m) & $d p$ \\
\hline 1 & 0,053 & 0,081 & 0,014 & 0,046 & 0,061 & 0,013 \\
\hline 2 & 0,063 & 0,156 & 0,025 & 0,002 & 0,093 & 0,017 \\
\hline 3 & 0,068 & 0,110 & 0,016 & 0,010 & 0,048 & 0,010 \\
\hline 4 & 0,060 & 0,132 & 0,020 & 0,052 & 0,103 & 0,017 \\
\hline 5 & 0,056 & 0,084 & 0,017 & 0,001 & 0,074 & 0,014 \\
\hline Média Grupo & $0,060 *$ & $0,113 *$ & 0,018 & $0,022 *$ & $0,076 *$ & 0,014 \\
\hline
\end{tabular}

$* \mathrm{p}<0,05$ diferença estatística.

$\mathrm{Na}$ condição olhos fechados, o equilíbrio corporal encontra-se sob orientação de apenas dois dos três sistemas aferentes, que fornecem feedback informando o sistema nervoso central acerca da posição e ajustes a serem realizados. Dessa forma, quando a visão está sendo suprimida, torna-se mais evidente a ação do sistema vestibular com o proprioceptivo (23). Os resultados apresentados vão ao encontro dos relatos na literatura de que o treino proprioceptivo pode aprimorar o equilibrio, favorecendo a estabilidade corporal através de menores deslocamentos do centro de pressão, e/ou com a sua recuperação de maneira mais rápida $(11,15,28)$. Lemos (15) afirma que, por meio da reeducação das reações de proteção obtidas através do treino proprioceptivo, o indivíduo consegue melhorar a recuperação do equilíbrio, retornando mais facilmente ao eixo normal da articulação em um curto espaço de tempo.

Estudos que averiguam a prevalência de lesões em atletas futebolistas apontam para a alta incidência de entorses em articulações como joelho e, principalmente, tornozelo $(8,3)$. O tornozelo é uma das articulações mais frequentemente lesadas porque atende a ambas as demandas - estabilidade e mobilidade. Contudo, se alguma de suas estruturas de suporte for lesada, essa articulação pode tornar-se bastante instável e comprometer o equilíbrio do atleta, levando a quedas e/ou a lesões mais graves como a entorse (3). A entorse constitui uma agressão ligamentar em decorrência da necessidade de manter o equilíbrio através da ampliação da base de suporte médio-lateral dos pés, utilizando para isso os limites anatômicos máximos e, em decorrência disso, estressando e lesionando essas estruturas $(6,17)$. A redução na amplitude de deslocamento médio-lateral do centro de pressão observado nas atletas deste estudo, após o treino proprioceptivo, demonstra um ganho no equilíbrio e estabilidade articular, especialmente em tornozelos. Isso contribui para a prevenção de lesões frequentes, como a entorse.

$\mathrm{Na}$ Tabela 4 observam-se as análises do COP na direção ântero-posterior (COPy) e na condição olhos fechados. Segundo a análise estatística, não houve diferença significativa nas variáveis (média, amplitude e desvio padrão) na comparação antes e após a aplicação do protocolo de propriocepção. Contudo, os resultados mostram uma tendência à diminuição para todas as variáveis analisadas após o treino proprioceptivo. Esse resultado sugere uma possível melhora na estabilidade da postura na direção ântero-posterior, assim como na médio-lateral, quando na ausência do feedback do sistema visual, e maior demanda do proprioceptivo no controle do equilíbrio.

Nos esportes de velocidade, como é o caso do futebol, é de suma importância que os receptores proprioceptivos estejam aptos a responder de forma breve e objetiva aos estímulos provenientes do movimento e/ou da postura. Os programas de treinamento proprioceptivo produzem uma diminuição no tempo da resposta muscular dos atletas que se tornam mais hábeis para um desempenho rápido e inesperado nos movimentos utilizados no esporte (18). Assim sendo, o sistema proprioceptivo estará "treinado" para reconhecer e responder mais rapidamente às situações de estresse, como uma situação de inversão, por exemplo, que são os principais mecanismos causadores de lesões. 
Tabela 4 - Dados refentes aos deslocamentos ântero-posteriores do centro de pressão (COPy) na condição - olhos fechados e apoio unipodal antes e após intervenção

\begin{tabular}{|c|c|c|c|c|c|c|}
\hline \multirow[b]{2}{*}{ Sujeito } & \multicolumn{3}{|c|}{ Pré-intervenção } & \multicolumn{3}{|c|}{ Pós-intervenção } \\
\hline & Média (m) & Amplitude (m) & $\mathrm{dp}$ & Média (m) & Amplitude (m) & $\mathrm{dp}$ \\
\hline 1 & 0,005 & 0,007 & 0,009 & 0,030 & 0,039 & 0,009 \\
\hline 2 & 0,014 & 0,050 & 0,009 & 0,009 & 0,040 & 0,010 \\
\hline 3 & 0,016 & 0,061 & 0,010 & 0,004 & 0,031 & 0,006 \\
\hline 4 & 0,023 & 0,103 & 0,020 & 0,020 & 0,041 & 0,008 \\
\hline 5 & 0,011 & 0,036 & 0,009 & 0,004 & 0,052 & 0,009 \\
\hline Média Grupo & 0,014 & 0,051 & 0,011 & 0,013 & 0,041 & 0,009 \\
\hline
\end{tabular}

Sheth et al. (12) afirmam que um treinamento proprioceptivo proporciona também maior estabilidade no tornozelo e maior economia no consumo de oxigênio e de energia por esses músculos, podendo melhorar assim o desempenho em atividades esportivas. Dessa forma, o treino proprioceptivo é um importante método de preparação do atleta, com o objetivo fundamental, que é a prevenção de lesões ou recidivas, e secundariamente, a melhora do desempenho motor.

O estudo de Mandelbaun (14), a partir da utilização de um programa de treinamento proprioceptivo em jogadoras de futebol, observou a redução no índice de lesões do ligamento cruzado anterior do joelho decorrentes de lesões em 88\% no primeiro ano, e de 74\% no segundo ano de aplicação do treino proprioceptivo, quando comparado ao grupo controle. Segundo o autor, tal resultado deve-se ao trabalho proprioceptivo, que contribui para a prevenção das lesões de joelho.

De maneira semelhante, Mohammadi (16) avaliou 80 atletas masculinos de futebol com histórico de inversão de tornozelos. Os sujeitos foram divididos em três grupos para participar de programas preventivos de recidiva de lesões. Os protocolos em cada grupo foram: proprioceptivo, de força, com uso de órteses, além de um grupo controle. Os resultados mostraram que apenas o treino proprioceptivo diferiu do grupo controle, reduzindo a frequência das inversões da articulação e demonstrando um aumento da força nos músculos, melhor controle postural e percepção corporal. Os achados dos referidos estudos reafirmam a importância desse treino na prevenção das lesões decorrentes da prática desse esporte $(14,16)$.

A Tabela 5 apresenta os dados referentes às oscilações laterais das participantes na condição olhos abertos. Observa-se um decréscimo, embora não significativo estatisticamente, nos valores da média e amplitude de oscilação do grupo quando comparado os dados pré e pós-intervenção. Esses resultados sugerem menor necessidade de deslocamento do COP para um efetivo controle neuromuscular, responsável pela manutenção da postura.

Quando o sistema nervoso central dispõe da aferência do sistema visual, vestibular e proprioceptivo para realizar o controle do equilíbrio, existe uma prevalência dos sinais visuais sobre os outros dois, para informar sobre o equilíbrio (29). É por esse motivo que uma pessoa consegue manter razoavelmente bem o equilíbrio, mesmo com a destruição do aparelho vestibular e dos receptores proprioceptivos (29). Na realização do teste na condição olhos abertos, é possível que o controle do equilíbrio tenha sido efetuado principalmente pelo feedback do sistema visual, em detrimento do proprioceptivo, não revelando, portanto, a possível melhora das respostas proprioceptivas após o treino proposto.

A falta de significância estatística nestes resultados também pode estar relacionada com o pequeno número de sujeitos participantes do grupo de estudo e com o número de sessões de prática do treinamento, 
que podem não ter sido suficientes para apresentar resultados significativos na melhora do controle do equilíbrio na condição olhos abertos.

Os dados apresentados na Tabela 6, referentes aos deslocamentos ântero-posterior na condição olhos abertos, não apresentaram diferenças estatisticamente significativas entre os valores pré e pós-intervenção. Não houve redução dos valores pré e pós-intervenção, como esperado pelos pesquisadores. Esse resultado pode ser justificado pela influência do sistema visual sobre o proprioceptivo, corroborando com a literatura que afirma que, diante da presença dos três sistemas aferentes de feedback (visual, proprioceptivo e vestibular), o sistema visual mantém a dominância sobre os demais $(23,29)$, não permitindo avaliar adequadamente as possíveis mudanças ocorridas no sistema proprioceptivo após a intervenção proposta.

Tabela 5 - Dados refentes aos deslocamentos médio-laterais do centro de pressão (COPx) na condição - olhos abertos e apoio unipodal antes e após intervenção

\begin{tabular}{|c|c|c|c|c|c|c|}
\hline \multirow[b]{2}{*}{ Sujeito } & \multicolumn{3}{|c|}{ Pré-intervenção } & \multicolumn{3}{|c|}{ Pós-intervenção } \\
\hline & Média (m) & Amplitude (m) & $\mathrm{dp}$ & Média (m) & Amplitude (m) & $\mathrm{dp}$ \\
\hline 1 & 0,054 & 0,039 & 0,007 & 0,057 & 0,035 & 0,007 \\
\hline 2 & 0,073 & 0,051 & 0,010 & 0,001 & 0,044 & 0,009 \\
\hline 3 & 0,054 & 0,038 & 0,006 & 0,001 & 0,028 & 0,007 \\
\hline 4 & 0,058 & 0,056 & 0,009 & 0,064 & 0,040 & 0,008 \\
\hline 5 & 0,046 & 0,036 & 0,008 & 0,008 & 0,051 & 0,009 \\
\hline Média Grupo & 0,058 & 0,044 & 0,008 & 0,026 & 0,040 & 0,008 \\
\hline
\end{tabular}

Tabela 6 - Dados refentes aos deslocamentos ântero-posteriores do centro de pressão (COPy) na condição - olhos abertos e apoio unipodal antes e após intervenção

\begin{tabular}{|c|c|c|c|c|c|c|}
\hline \multirow[b]{2}{*}{ Sujeito } & \multicolumn{3}{|c|}{ Pré-intervenção } & \multicolumn{3}{|c|}{ Pós-intervenção } \\
\hline & Média (m) & Amplitude (m) & $\mathrm{dp}$ & Média (m) & Amplitude (m) & $\mathrm{dp}$ \\
\hline 1 & 0,002 & 0,002 & 0,008 & 0,042 & 0,025 & 0,007 \\
\hline 2 & 0,002 & 0,024 & 0,006 & 0,001 & 0,036 & 0,008 \\
\hline 3 & 0,040 & 0,032 & 0,009 & 0,007 & 0,016 & 0,004 \\
\hline 4 & 0,013 & 0,026 & 0,008 & 0,018 & 0,039 & 0,010 \\
\hline 5 & 0,001 & 0,016 & 0,003 & 0,025 & 0,049 & 0,003 \\
\hline Média Grupo & 0,004 & 0,019 & 0,007 & 0,019 & 0,027 & 0,006 \\
\hline
\end{tabular}




\section{Considerações finais}

Os achados do presente estudo podem ser sistematizados nos seguintes aspectos:

a) o pouco conhecimento sobre a propriocepção pelas atletas participantes do estudo demonstra que o treino proprioceptivo não era incorporado efetivamente nas rotinas de treinamento desportivo;

b) o treinamento proprioceptivo é um método efetivo para melhorar o equilíbrio e a estabilidade postural, principalmente no controle médio lateral (COPx);

c) a ausência de resultados significativos no controle do equilíbrio na condição olhos abertos pode estar relacionada à predominância do sistema visual como fonte preferencial de aferência utilizada pelo sistema nervoso central no controle do equilíbrio, dificultando a observação dos progressos no equilíbrio efetuado pelo treino do sistema proprioceptivo;

d) o treino proprioceptivo favorece tanto a melhora do controle do equilíbrio, quanto a prevenção de lesões corporais dos praticantes.

Em vistas desses achados, sugere-se que protocolos proprioceptivos sejam aplicados durante período e amostras maiores, com participação de grupo controle, para que se possa futuramente padronizar períodos e técnicas mais efetivas para este tipo treinamento.

\section{Referências}

1. Santana WC, Reis HHB. Futsal feminino: perfil e implicações pedagógicas. Rev Bras Ciênc Mov. 2003; 11(4):45-50.

2. Goellner SV. Mulheres e futebol no Brasil. Rev Bras Educ Fís Esporte. 2005;19(2):143-51.

3. Cohen M, Abdalla RJ, Ejnisman B, Amaro JT. Lesões ortopédicas no futebol. Rev Bras Ortopedia. 1997;32(12): 40-4.

4. Stewien ETM, Camargo OPA. Ocorrência de entorse e lesões do joelho em jogadores de futebol da cidade de Manaus, Amazonas. Acta Ortop Bras. 2005;13(3):141-6.

5. Ribeiro CZP, Akashi PMH, Sacco ICN, Pedrinelli A. Relação entre alterações posturais e lesões do aparelho locomotor em atletas de futebol de salão. Rev Bras Med Esporte. 2003;9(2):91-97.

6. Simões NVN. Lesões desportivas em praticantes de atividade física: uma revisão bibliográfica. Rev Bras Fisioter. 2005;9(2):123-8.

7. Bonetti LV, Coelho R. Prevenção de lesões desportivas mais freqüentes na prática do futsal [monografia conclusão de curso]. Santa Maria: Universidade Federal de Santa Maria; 2004.

8. Salomão O, Carvalho Neto J, Traldi IH, Fernandes TD, Carvalho Jr AE. Tratamento da lesão ligamentar aguda do tornozelo em atletas. Rev Bras Ortop. 1996;31(3):253-6.

9. Tookuni KS, Neto RB, Pereira CAM, Souza DR, Greve JMA, Ayala AA. Análise comparativa do controle postural de indivíduos com e sem lesão do ligamento cruzado anterior do joelho. Acta Ortop Bras. 2005;13(3):115-9.

10. Winter DA. Human balance and posture control during standing and walking. Gait \& Posture. 1995;3(4):193-214.

11. Silvestre MV, Lima WC. Importância do treinamento proprioceptivo na reabilitação de entorse de tornozelo. Fisioter Mov. 2003;16(2):27-34. 
12. Sheth P, Yu B, Laskowsk ER, An K. Ankle disk training influences reaction times of selected muscles in a simulated ankle sprain. Am J Sports Med. 1997;25(4):538-43.

13. Bahr R, Evert V, Alllard VB, Jos T, Lex B, Willem M. The effect of a propioceptive balance board training program for the prevention of ankle sprains. Am J Sports Med. 2004;32(6):1385-93.

14. Mandelbaum BR, Silvers HJ, Watanabe DS, Knarr JF, Thomas SD, Griffin LY, et al. Effectiveness of a neuromuscular and proprioceptive training program in preventing anterior cruciate ligament injuries in female athletes: 2-Year Follow-up. Am J Sports Med. 2005;33(7):1003-10.

15. Lemos JC. Exercícios proprioceptivos em lesões de joelho. Fisioter Mov. 1992;5(1):53-60.

16. Mohammadi F. Comparison of 3 preventive methods to reduce the recurrence of ankle inversion sprains in male soccer players. Am J Sports Med 2007;35:922-932.

17. Renstrom PAFH, Lynch SA. Lesões ligamentares do tornozelo. Rev Bras Med Esporte. 1999;5(1):13-23.

18. Araújo ADS, Merlo JRC, Moreira C. Reeducação neuromuscular e proprioceptiva em pacientes submetidos à reconstrução do ligamento cruzado anterior. Rev Fisioter Brasil. 2003;4(3):217-21.

19. Hawkins RD, Hulse Ma, Wilkinson C, Hodson A, Gibson M. The association football medical research programme: an audit of injuriesin professional football. Br J Sports Med. 2001;35(1):43-7.

20. Diener HC, Dichgans J, Bruzek W, Selinka H. Stabilization of human posture during induced oscillations of the body. Exp Brain Res. 1982;45(1-2):126-32.

21. LêClair K, Riach C. Postural stability measures: what to measure and for how long. Clin Biomech. 1996;11(3):176-178.

22. Vuillerme N, Nougier V, Teasdale N. Effect of a reaction time task on postural control in humans. Neurosci Lett. 2000;291(2):77-80.

23. Latash ML, Ferreira SS, Wieczrek SA, Duarte M. Movement sway: changes in postural voluntary shifts of the center of pressure. Exp Brain Res. 2003;150(3):314-24.

24. Winter DA. Biomechanics and motor control of human movement. New Jersey: Copyright; 2004.

25. Salgado ASI. Fisioterapia: reeducação proprioceptiva do joelho e tornozelo. São Paulo: Lovise; 1995.

26. Prentice WE. Técnicas em reabilitação músculo-esquelética. São Paulo: Artmed; 2003.

27. Kisner C, Colby LA. Exercícios terapêuticos: fundamentos e técnicas. São Paulo: 4. ed. Manolle; 2003.

28. Bonfim TR, Barela JA. Efeito da manipulação da informação sensorial na propriocepção e no controle postural. Fisioter Mov. 2007;20(2):107-17.

29. Shumway-Cook A, Woollacott M. Motor control: theory and pratical applications. Baltimore: Williams \& Wilkins; 1995.

Recebido: 19/07/2009

Received: 07/19/2009

Aprovado: 24/03/2010

Approved: 03/24/2010

Revisado: 03/05/2010

Reviewed: 05/03/2010 